\title{
Coherent Cooperative Transmission From Multiple Adjacent Antennas To a Distant Stationary Antenna Through AWGN Channels
}

\author{
Yung-Szu Tu and Gregory J. Pottie \\ Electrical Engineering Department \\ University of California \\ Los Angeles, CA 90095-1594 \\ \{yungszu,pottie\}@ee.ucla.edu
}

\begin{abstract}
The information transmission from a cluster of adjacent antennas to a distant stationary antenna through additive white Gaussian noise channels is considered. With the coherent cooperative transmission approach, information bits are first shared among this cluster of transmit antennas, and then these antennas adjust their carrier phases, carrier frequencies, and symbol timings, so that signals containing the same data arrive at the receive antenna coherently and thus combine constructively in the transmission medium. To achieve this, first, a common time scale must be tracked by all transmit antennas. This time scale is distributed in a master-slave style, with the clock of the receive antenna acting as the master clock. Secondly, due to the different propagation delays both from the master to the slaves, and from the transmit antenna to the receive antennas, pre-compensation is employed at transmit antennas. We derive a mathematical model for this transmission scheme. The probability density functions of variables in this model are determined, and the bit error rates are computed, for various data energy distributions and headers.
\end{abstract}

\section{INTRODUCTION}

$\mathrm{I}_{\mathrm{t}}^{\mathrm{N}}$ N THE context of fading channels, the use of multiple antennas has been demonstrated to provide antenna diversity (also referred to as space diversity), which dramatically enhances system performance and channel capacity[1][2][3]. Compared with additive white Gaussian noise (AWGN) channels, the problem with fading channels, in terms of error probability, comes from the extra random attenuation of the path gain. When the channel is in deep fading, the path gain declines and consequently the error probability deteriorates given the same noise level. When the error probability is averaged over the whole possible path gain, the poor performance at deep fading dominates. With multiple antennas, we can take advantage of the randomness of the fading. The likelihood that all channels are in deep fading is less than the likelihood that a single channel is in deep fading. In other words, if one channel is in deep fading, the information may still be conveyed through other channels.

The usage of multiple transmit antennas is also preferred and available in future planetary exploration or terrestrial monitoring, where it is desired to deploy large numbers of ground sensors. The information collected from all sensors often has to be brought together at a hub. Two sensors that are far from each other may also need to exchange information. Due to the distance-squared wave propagation loss, communication through multiple short-distance hops is preferable to a single long-distance hop. However, the situation may be that these relays are not present, and there are several sensors in the vicinity of the information source. For example, a Mars orbiter may be chosen as the hub. The altitude of the orbiter is on the order of hundred kilometers, the distance between sensors may be in the order of kilometers, but there is no relay between the orbiter and the Mars surface. Here, communication cost between sensors is insignificant, compared with communication between sensors and the orbiter. In cooperative communications, information is first shared among a cluster of neighboring sensors and then sensors in this cluster cooperatively convey the information to the destination, in this example the orbiter, using all resources for these sensors to achieve lower error probability or higher data rate. Among all resources, the energy is of the most concern. The reason is there is no infrastructure to provide energy to sensors, while energy contained in sensors is limited because the size of sensors is small. On the other hand, transmission from the satellite is less constrained as solar energy can be more cheaply generated. Therefore, the most challenging link is from the multiple sensors to the satellite.

For this situation, we consider AWGN channels. Line of sight (LOS) paths exist between sensors and the satellite, and there are few scatterers. Under this assumption, the transmit diversity techniques can still apply but the improvement is not as substantial as for fading channels because these techniques are designed in view of the shortcomings of fading channels.

Another difference is the volatility of channels. Among these techniques, channel state information (CSI) is estimated by receive antennas, which are the satellites in our example, and used for detection and decoding. However, CSI is not fed back to the transmit antennas, which are the sensors in our example, for the purpose of coding and modulation. If the channel is changing fast, it is indeed useless to feed back CSI, adaptively code and modulate. Since we consider AWGN channels, the only CSI variation comes from the distance between transmit and receive antennas, and we assume the channel changes so slowly that the transmit antennas can exploit the CSI feedback.

\section{COHERENT COOPERATIVE TRANSMISSION}

Herein, we focus on the single receive antenna situation. One technique to exploit energy stored in separate transmit antennas is to adjust all EM waves so that waves emitted from each transmit antenna combine coherently at the receive antenna. Fig. 1 illustrates this wave addition for the setting of two transmit antennas. If the waves are of the same amplitude 


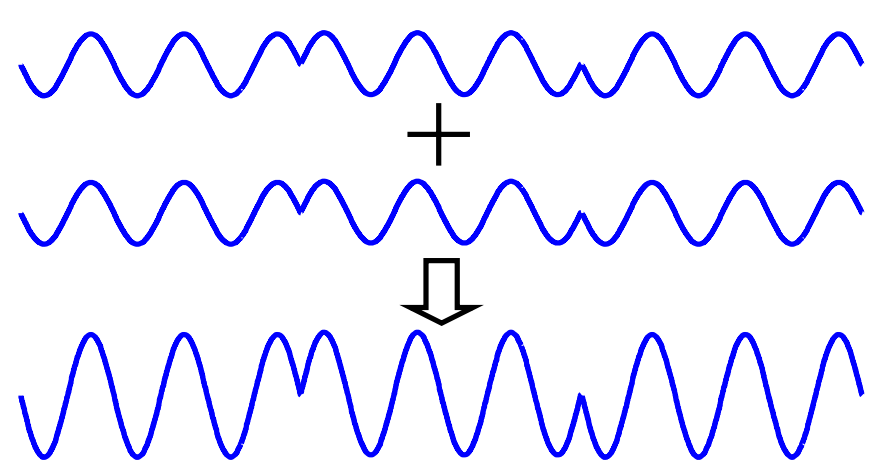

Fig. 1. EM waves from two transmit antennas combine coherently at the receive antenna.

and perfectly coherent, the amplitude of the received wave is twice the amplitude of each component wave.

This leads to increased channel capacity. For the AWGN channel, the channel capacity is

$$
C=W \log \left(1+P_{a v} /\left(W N_{0}\right)\right),
$$

where $W$ is the bandwidth in Hertz, $N_{0}$ is the one-sided power spectral density, and $P_{a v}$ is the average received power. When each antenna transmits its own information independently, $P_{a v}$ is equal to the summation of each transmission power times individual attenuation. Assuming identical attenuation $a$, identical transmission power $P_{t x}$, and $N$ transmit antennas, then $P_{a v}$ is equal to $a N P_{t x}$. In contrast, if signals combine coherently

$$
P_{a v}=\left(N \sqrt{a P_{t x}}\right)^{2}=a N^{2} P_{t x},
$$

which increases with the square of the number of transmit antennas.

\section{SYSTEM ARCHITECTURE}

To achieve this, the received carrier frequencies, the carrier phases, and the symbol timings of all EM waves have to be the same when received by the receive antenna. As clocks are triggered by oscillators, the instantaneous phase of the oscillator of an antenna can be precisely determined by the time scale at that antenna. There are two network synchronization approaches [5] to be considered: mutual synchronization and master-slave synchronization . We will discuss which approach is better for our purpose. In addition, pre-compensation for the trip delays must be incorporated at transmit antennas.

The master-slave synchronization fits our objective better than the mutual one. With mutual synchronization, each clock collaborates with other clocks to determine the common time scale. This approach is preferred when no clock is superior to others and the robustness of the common time scale, with respect to the drift of any clock, is very crucial. However, there are two drawbacks. First, considerable overhead, which consumes energy, is required for clocks to determine the common clock scale. Secondly, a multiple access scheme must be employed to differentiate one clock from the other. Our goal is to align all EM waves coherently at the receive antenna with little extra energy dissipation. Whether the common time scale is robust does not directly relate to the energy dissipation. Thus, we choose the master-slave synchronization approach. Once each slave clock keeps track of the variation of the master clock well, the clock drift is not an issue. The question of which antenna is the appropriate master will be discussed later.

Pre-compensation is required for each transmit antenna to compensate unequal propagation delays. In the area of network synchronization, the propagation delays from the master clock to the slave clocks are compensated either in the master clock in advance or in the slave clocks afterward, so that at any point in time, all clocks have the same time scale. However, our objective is more than that. We desire all waves to arrive at the receive antenna coherently. This is similar to the time requirements for the time division multiple access in satellite communications, where signals from transmit antennas must arrive at the satellite at specified time points. If the delays from all transmit antennas to the receive antenna were the same, the consequence was just a time shift of the time scale, and all signals could arrive coherently. However, because the delays are actually different, clocks in transmit antennas have to be pre-compensated to account for different propagation delays from the transmit antennas to the receive antenna. Thus, the delays from the master to the slave clocks have to be compensated, and the delays from the transmit antennas to the receive antenna must be pre-compensated.

Two types of the combination of master-slave synchronization and pre-compensation have been investigated in [5]. The first type is the open-loop approach. For a master-slave pair, both master and slave antennas transmit their clock scales to each other. Based on the clock scales of the incoming wave and the local antenna, the clock difference and pre-compensation are constantly calculated cooperatively by the master and the slave antenna. The slave clock accordingly updates its clock and pre-compensation by changing delay, while the master updated the pre-compensation only. The slave clock makes no effort to adjust its oscillator frequency in response to the clock difference. If the master and the slave oscillator frequencies are off by a large amount, the clock difference will increase rapidly after updates, and this will result in poor synchronization. The second type is the closed-loop approach. The slave clock is a voltage-controlled oscillator (VCO). The error signal is the clock difference mentioned above, and this signal is used to adjust the VCO frequency, rather the delay as in the open-loop approach. The problems with this approach are the stability and the tracking ability. Unlike ordinary phase lock loops, this loop includes two significant delays, one in the master-to-slave transmission and the other in the slave-to-master transmission. Because the delay is so huge, to keep the loop stable, the loop bandwidth must be narrow, and the tracking ability will be reduced.

Our approach is illustrated in Fig. 2. It employs a VCO at the slave antenna to track the master clock frequency which is received at the slave antenna, and the phase comparison is done locally. Thus, we avoid the problem due to long propagation delay as in the closed-loop approach, while slave clocks can track the frequency of the master clock. 


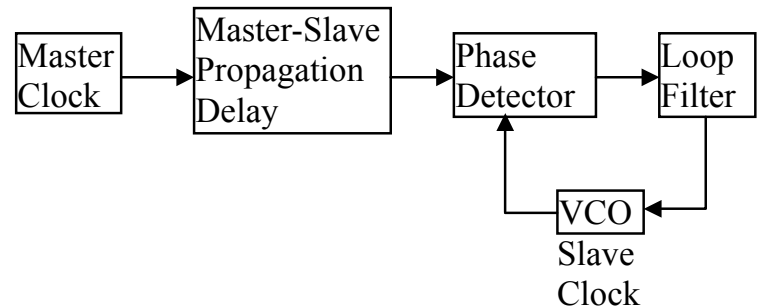

Fig. 2. The diagram of our master-slave synchronization.

Compensation for the master-slave propagation delay is implemented jointly with pre-compensation for transmit-receive propagation delay, as illustrated in Fig. 3. If we wanted to equalize all time scales, we would have to compensate slave clocks for the master-slave propagation delay with delay elements, and this would require a multiple access scheme among slave antennas and a lot of signaling to determine the amount of compensation. Since what matters is whether waves arrive at the receive antenna coherently, this process can be combined with transmit-receive propagation delay pre-compensation. Note the clock of the transmit antenna can be a master or a slave clock. The time difference measured at the receive antenna is the sum of both propagation delays, and they are indistinguishable. This value is modulated, fed back, demodulated, and used in the single delay element. We assume the receive antenna is moving slowly so that it can be viewed stationary during the interval between pre-compensation estimation and coherent combining, while the value of pre-compensation has to be estimated and updated periodically. The new estimate is based upon the signal which is delayed by the previous pre-compensation estimate. Thus, this new estimate can be considered the adjustment to the previous estimate.

We choose the master clock based on the criterion of low energy dissipation. The information for pre-compensation has to be modulated by the oscillator of the receive antenna, and fed back to the transmit antenna. The clock of the receive antenna can act as the master clock without consuming any extra energy since the time scale can be embedded in the carrier phase and the symbol boundary. In order to extract the time scale, the slave clocks have to remove the information sequence[6].

Based on the preceding discussion, the block diagrams of the receive antenna with the master clock and the transmit antenna with the slave clock are illustrated in Fig. 4 and Fig. 5. The double line represents in-phase and quadrature components.

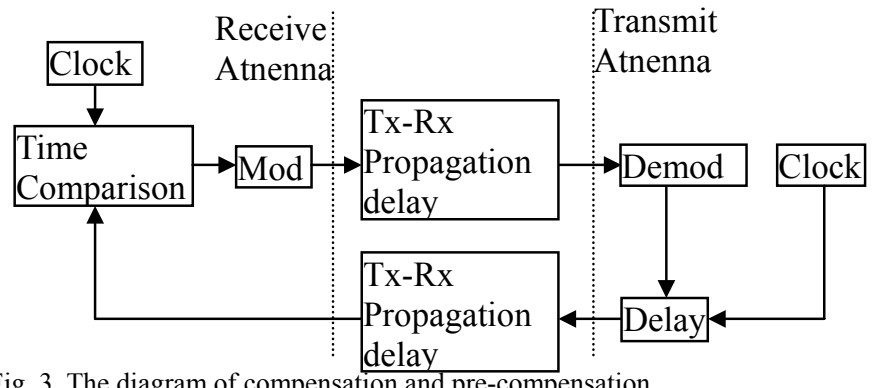

Fig. 3. The diagram of compensation and pre-compensation
The time scale of the master clock is conveyed through a pseudo-noise ( $\mathrm{PN})$ sequence and the phase embedded in the continuous sinusoidal carrier. They work in a complementary way. The phase of the sine wave changes faster than the PN sequence. Thus, it can provide more precise timing. However, there is a severe $2 p$ ambiguity problem. The period of the PN sequence is much longer, so it can solve this ambiguity. On the other hand, since the chip rate is lower than the frequency of the sinusoidal carrier, the timing resolution is limited. By multiplying the PN sequence by the sine wave, precise time scale without ambiguity can be realized and transmitted to all slave clocks. Each slave clock uses a phase lock loop and a delay lock loop to maintain synchronism with the carrier and the PN sequence respectively.

In order to estimate the individual pre-compensation values for each transmit antenna, every transmit antenna has to transmit its time scale back to the satellite. We also use the combination of PN sequence and sinusoidal carrier to represent the time scale. To avoid interfering with the frequency band occupied by the downlink, i.e. from the master to the slave clocks, the carrier frequency of this synchronization signal is shifted to another frequency band, while this new carrier must be derived from the retrieved carrier so that the feedback signal can represent the retrieved time scale. To achieve this, a frequency multiplier or a divider is inserted after the output of the PLL. Additionally, the receive antenna must be able to differentiate synchronization signals from different transmit antennas. In other words, a specific multiple access scheme must be employed. DS-CDMA and TDMA are good choices for this purpose. The advantage of these two schemes over FDMA is that all transmit antennas use the same carrier frequency, which is required in the coherent transmission. Therefore, the phase pre-compensation value can be used directly in coherent transmission without any conversion. We use DS-CDMA in Fig. 4 and Fig. 5 as illustration because we would like to point out a crucial point for DS-CDMA. There must be two PN sequences multiplied together, just like in IS-95[7]. If only one sequence was used, since the different propagation delay affects the phase of received PN sequence, there would be no way to distinguish transmit antennas. Thus, both long PN and short PN sequences are employed.

At each transmit antenna, both information and synchroni-

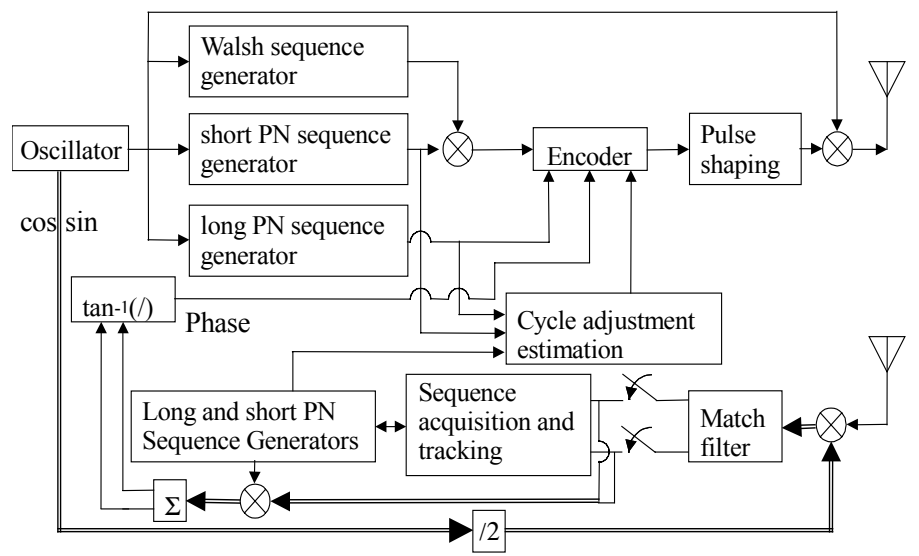

Fig. 4. The block diagram of the receive antenna with the master clock. 


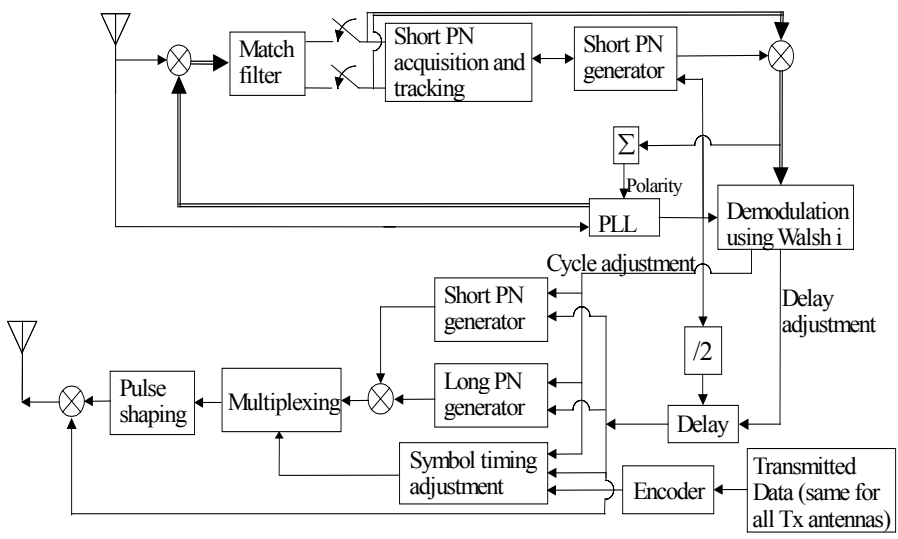

Fig. 5. The block diagram of the transmit antenna with the slave clock.

zation signals are transmitted. These two kinds of signals are multiplexed in the time or code domain, while the timing and phase reference are shared. Data signals from all transmit antennas occupy the same channel in the time or code domain. Because of the operations described above, information signals are received at the receive antenna coherently.

\section{Mathematical Model}

Assuming the information signal and the synchronization signals are time multiplexed, at the receive antenna, the received information signals from all transmit antennas are combined as

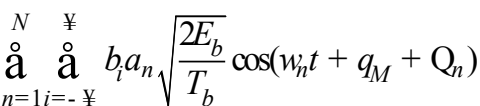

$$
\begin{aligned}
& \aleph_{(}\left(t-i T_{b}+\left(k_{n} T_{w}+\mathrm{Q}_{n} / w_{n}\right)\right),
\end{aligned}
$$

where $b_{i}= \pm 1$ is the information bit, $a_{n}$ is the attenuation, $E_{b}$ is the bit energy, $w_{n}$ is the carrier frequency, $q_{M}$ is the phase of the master oscillator, $\mathrm{Q}_{n}$ is the phase offset and also a random process, $h(t)$ is the pulse, $T_{b}$ is the symbol duration, $k_{n}$ is an integer, and $T_{w}$ is the period of the carrier. $k_{n} T_{w}+\mathrm{Q}_{n} / w_{n}$ represents the time offset. It is assumed $a_{n}=1 " n, w_{n}=w n$ due to the operation of PLL, $k_{n} \gg k \quad n$ which means the chip offset estimation and pre-compensation track the channel dynamics very well. $\mathrm{Q}_{n} / w_{n}$ is insignificant, compared with the support of $h(t)$. Thus, $h\left(t-i T_{s}+\left(k_{n} T_{w}+\mathrm{Q}_{n} / w_{n}\right)\right)$ is almost the same for all transmit antennas, at any point in time. Therefore, only $\cos \left(w_{n} t+\mathrm{Q}_{n}\right)$ is of concern.

\section{Performance ANALysis}

While the pre-compensation estimate is update periodically, we assume that the propagation delays are constant during the interval from when they are estimated to when signals are coherently combined in the receive antenna. We assume all transmit antennas are independent from each other. Thus, $\mathrm{Q}_{n}$ 's are independent identical distributed. The components in $\mathrm{Q}_{n}$ are determined by analyzing the procedures of coherent transmission, as listed in Table I. At time $t_{1}$, the phase of wave which the slave clock $n$ tracks is $q_{M}$ plus the phase offset $j_{n}$ due to the propagation delay. The slave clock bounces back the time scale with the PLL phase error $q_{e, n}\left(t_{1}\right)$ and the present pre-compensation estimate $q_{\text {est }}$. Owing to the propagation delay again, the phase receive by the receive antenna is further shifted by $j_{n}$ at time $t_{2}$. The receive antenna estimates the phase pre-compensation with phase error $f_{e, n}\left(t_{2}\right)$. The new estimate $q_{e s t}\left(t_{2}\right)$ is sent back to the transmit antenna $n$, and subtracted from the old estimate $q_{e s t}$. At time $t_{4}$, the transmit antenna transmits information data to be combined coherently with the master clock phase $q_{m}$, the phase offset $j_{n}$, the PLL phase error $q_{e, n}\left(t_{4}\right)$, and the updated pre-compensation $q_{\text {est }}-q_{\text {est }}\left(t_{2}\right)$. Therefore, the wave arrives at the receive antenna with phase

$$
\begin{aligned}
& q_{M}+2 j_{n}+q_{e, n}\left(t_{4}\right)+q_{e s t}-q_{e s t}\left(t_{2}\right) \\
& =q_{M}+q_{e, n}\left(t_{4}\right)-q_{e, n}\left(t_{1}\right)+f_{e, n}\left(t_{2}\right) .
\end{aligned}
$$

The probability density function (pdf) of $q_{e, n}(y$ is derived in [8] by solving the Fokker-Planck equation

$$
P\left(q_{e, n}\right)=\frac{\exp \left(h \cos \left(q_{e, n}\right)\right)}{2 p I_{0}(h)},
$$

where $h$ is the signal to noise ratio in the phase lock loop. Assuming $q_{e, n}\left(t_{4}\right)$ and $q_{e, n}\left(t_{1}\right)$ are independent, the pdf of

\begin{tabular}{|c|c|c|c|}
\hline Time & Action & Receive Antenna & Transmit Antenna $n$ \\
\hline always & $\begin{array}{l}\text { The master clock } \\
\text { transmits the time } \\
\text { scale }\end{array}$ & $\cos \left(w_{M} t+q_{M}\right)$ & \\
\hline$t_{1}$ & $\begin{array}{l}\text { The slave clock } n \\
\text { receives the time } \\
\text { scale }\end{array}$ & & $\begin{array}{l}\cos \left(w t+q_{M}\right. \\
\left.+j_{n}\right)\end{array}$ \\
\hline$t_{1}$ & $\begin{array}{l}\text { The slave clock } n \\
\text { bounces back the } \\
\text { time scale }\end{array}$ & & $\begin{array}{l}\cos \left(w t+q_{M}+j_{n}\right. \\
\left.+q_{e, n}\left(t_{1}\right)+q_{e s t}\right)\end{array}$ \\
\hline$t_{2}$ & $\begin{array}{l}\text { The receive an- } \\
\text { tenna (master } \\
\text { clock) receives } \\
\text { the bounce }\end{array}$ & $\begin{array}{l}\cos \left(w t+q_{M}\right. \\
+j_{n}+q_{e, n}\left(t_{1}\right) \\
\left.+q_{e s t}+j_{n}\right)\end{array}$ & \\
\hline$t_{2}$ & $\begin{array}{l}\text { The receive an- } \\
\text { tenna estimates } \\
\text { the new phase } \\
\text { pre-compensation }\end{array}$ & $\begin{array}{l}q_{e s t}\left(t_{2}\right)=2 j_{n} \\
+q_{e, n}\left(t_{1}\right)+q_{e s t} \\
+f_{e, n}\left(t_{2}\right)\end{array}$ & \\
\hline$t_{3}$ & $\begin{array}{l}\text { The receive an- } \\
\text { tenna feeds back } \\
\text { the estimate }\end{array}$ & & \\
\hline$t_{4}$ & $\begin{array}{l}\text { The transmit } \\
\text { antenna } n \text { (slave } \\
\text { clock) receives } \\
\text { the new estimate, } \\
\text { and transmit } \\
\text { information data }\end{array}$ & & $\begin{array}{l}\cos \left(w t+q_{M}\right. \\
+j_{n}+q_{e, n}\left(t_{4}\right) \\
\left.+q_{e s t}-q_{e s t}\left(t_{2}\right)\right)\end{array}$ \\
\hline$t_{5}$ & $\begin{array}{l}\text { Waves are co- } \\
\text { herently com- } \\
\text { bined, and re- } \\
\text { ceived by the } \\
\text { receive antenna }\end{array}$ & $\begin{array}{l}\cos \left(w t+q_{M}\right. \\
+2 j_{n}+q_{e, n}\left(t_{4}\right) \\
\left.+q_{e s t}-q_{e s t}\left(t_{2}\right)\right)\end{array}$ & \\
\hline
\end{tabular}
$q_{e, n}\left(t_{4}\right)-q_{e, n}\left(t_{1}\right)$ is

TABLE I

TIME FLOW OF COHERENT TRANSMISSION 


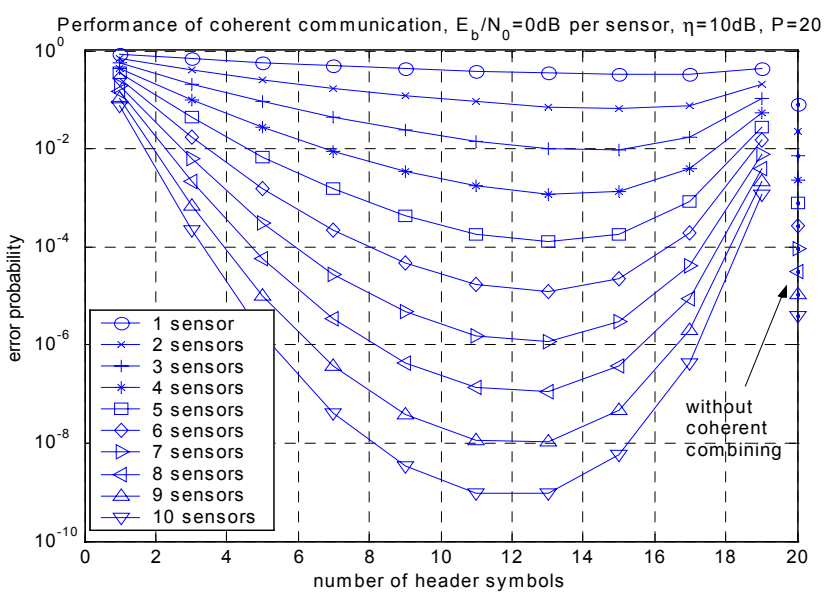

Fig. 7. Performance for various header sizes, stationary receive antenna.

$$
\begin{aligned}
& P\left(q_{e, n}\left(t_{4}\right)-q_{e, n}\left(t_{1}\right)\right) \\
& =\frac{I_{0}\left[\left|h+h \exp \left(j\left(q_{e, n}\left(t_{4}\right)-q_{e, n}\left(t_{1}\right)\right)\right)\right|\right]}{2 p\left[I_{0}(h)\right]^{2}},
\end{aligned}
$$

where $I_{0}(\ngtr$ is the first kind Bessel function with order 0 . The pdf of $f_{e, n}\left(t_{2}\right)$ is derived in [9].

To investigate the effect of energy distribution between information and synchronization signals, information data is divided into groups of $D$ symbols, and the synchronization signal, which is used for pre-compensation estimation and also called the header, is appended to form a packet of $P$ symbols. Then, the pdf of $f_{e, n}\left(t_{2}\right)$ can be written as

$$
\begin{aligned}
& P\left(f_{e, n}\right)=\frac{e^{-\frac{D E_{b} H}{P N_{o}}}}{2 p}+\sqrt{\frac{D E_{b} H}{p P N_{o}}} \cos \left(f_{e, n}\right) e^{\frac{D E_{b} H}{P N_{o}} \sin ^{2}\left(f_{e, n}\right)}
\end{aligned}
$$

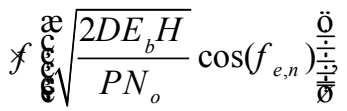

where $H=P-D, E_{b}$ is the total energy consumption per packet per transmit antenna divided by the number of information bits in a packet, $N_{0}$ is the one-sided noise spectrum density, and

$$
f(x)=\frac{1}{2 p} \underset{-\neq}{\stackrel{x}{\mathbf{O}}} e^{-\frac{w^{2}}{2}} d w .
$$

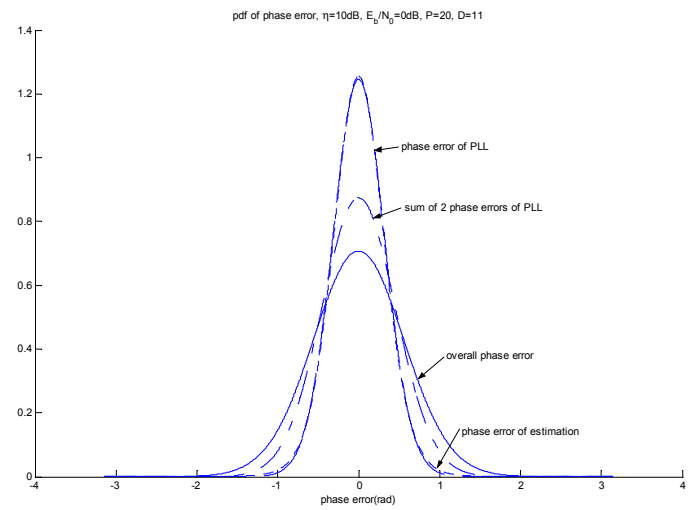

Fig. 6. The pdf's of several random variables.
Then, the pdf of $\mathrm{Q}_{n}$ is determined numerically. The pdf's of andom variables $q_{e, n}, q_{e, n}\left(t_{4}\right)-q_{e, n}\left(t_{1}\right), f_{e, n}$, and $\mathrm{Q}_{n}$ ire plotted in Fig. 6. The estimate is updated per packet. The ombined signal is demodulated at the receive antenna. Asuming BPSK is employed, the bit error rate for various leader sizes $H$ is depicted in Fig. 7. As more symbols are llocated for the header, the phase pre-compensation estimaion is more accurate, whereas less energy remains for infornation. The figure shows there is an optimum distribution of nergy for synchronization and for information transmission. Nhen compared with communication where all energy conumption is concentrated in a single transmit antenna, it is bvious that coherent cooperative transmission can achieve a ower bit error rate.

\section{CONCLUSION}

We propose a transmission scheme for the setting where transmit antennas are in the vicinity, while the single receive antenna is in the distance. By adjusting the carrier phase, frequencies, and symbol timings of transmit antennas, signals arrive at the receive antenna coherently, and thus combine constructively in the transmission medium. The adjustment is achieved by tracking the clock of the receive antenna, and pre-compensating transmission delays. Based on the mathematical model, we compute the system performance for the cases of the stationary and the non-stationary receive antenna. It is verified that this scheme can lower the error rate, compared with the situation where the same amount energy emanates from a single transmit antenna. An optimal energy distribution between information and synchronization is observed.

\section{REFERENCES}

[1] V. Tarokh, N. Seshadri, and A. R. Calderbank, "Space-time codes for high data rate wireless communications: performance criterion and code construction," IEEE Trans. Inform. Theory, vol. 44, pp. 744-765, Mar. 1998.

[2] J.-C. Guey, M. P. Fitz, M. R. Bell and W.-Y. Kuo, "Signal design for transmitter diversity wireless communication systems over Rayleigh fading channgles," in Proc. IEEE VTC'96, pp. 136-140.

[3] G. J. Foschini, Jr. and M. J. Gans, "On limits of wireless communication in a fading environment when using multiple antennas," Wireless Personal Commun, 6:311-335, Mar. 1998.

[4] W. C. Lindsey, F. Ghazvinian, W. C. Hagmann, and K. Dessouky, "Network synchronization," Proc. IEEE, vol. 73, pp. 1445-1467, Oct. 1985.

[5] W. C. Hagmann, "Network synchronization techniques for satellite communication systems," Ph.D. dissertation, USC, Los Angeles, CA, 1981.

[6] J. G. Proakis, Digital Communications, 4th ed., New York:McGraw-Hill, 2000.

[7] J. S. Lee and L. E. Miller, CDMA systems engineering handbook. Boston: Arch House, 1998.

[8] A. J. Viterbi, Principles of Coherent Communication. New York: McGraw-Hill, 1966.

[9] K. Quirk, "The Effects of Estimation Errors on Direct Sequence Spread Spectrum Receiver Performance," Ph.D. disserattion, University of California, San Diego, CA, 2000. 